\title{
Right ventricular failure following left ventricular assist device implantation is associated with a preoperative pro- inflammatory response
}

Paul C. Tang ${ }^{1 *}$, Jonathan W. Haft ${ }^{1}$, Matthew A. Romano ${ }^{1}$, Abbas Bitar², Reema Hasan², Maryse Palardy², Keith D. Aaronson ${ }^{2}$ and Francis D. Pagani ${ }^{1}$

\begin{abstract}
Background: Systemic inflammation during implant of a durable left ventricular assist device (LVAD) may contribute to adverse outcomes. We investigated the association of the preoperative inflammatory markers with subsequent right ventricular failure (RVF).

Materials and methods: Prospective data was collected on 489 patients from 2003 through 2017 who underwent implantation of a durable LVAD. Uni- and multivariable correlation with leukocytosis was determined using linear and binary logistic regression. The population was also separated into low $(<10.5 \mathrm{~K} / \mathrm{ul}, n=362)$ and high $(\geq 10.5 \mathrm{~K} / \mathrm{ul}, n=127)$ white blood cell count (WBC) groups. Mantel-Cox statistics was used to analyze survival data.

Results: Postop RVF was associated with a higher preop WBC (11.3 \pm 5.7 vs $8.7 \pm 3.1)$ and C-reactive protein (CRP, $5.6 \pm 4.4$ vs $3.3 \pm 4.7)$ levels. Multivariable analysis identified an independent association between increased WBC preoperatively with increased lactate dehydrogenase $(\mathrm{LDH}, P<0.001)$, heart rate $(P<0.001)$, CRP $(P=0.006)$, creatinine $(P=0.048)$, and INR $(P=0$. 049). The high WBC group was more likely to be on preoperative temporary circulatory support (17.3\% vs $6.4 \%, P<0.001)$ with a trend towards greater use of an intra-aortic balloon pump (55.9\% vs $47.2 \%, P=0.093)$. The high WBC group had poorer mid-term survival $(P=0.042)$.

Conclusions: Postop RVF is associated with a preoperative pro-inflammatory environment. This may be secondary to the increased systemic stress of decompensated heart failure. Systemic inflammation in the decompensated heart failure may contribute to RVF after LVAD implant.
\end{abstract}

Keywords: Inflammation, Left ventricular assist device, Right ventricular failure, Heart failure

\section{Introduction}

Inflammatory mediators are known to contribute to the development and progression of heart failure $[1,2]$ In the setting of durable left ventricular assist device (LVAD) implantation, postoperative right ventricular failure (RVF) complicates $9-44 \%$ of implants. This is associated with a greater incidence of morbidity, decreased

\footnotetext{
* Correspondence: tangpaul@med.umich.edu

Meeting Presentation: Abstract presentation at the International Society For Heart \& Lung Transplantation 38 ${ }^{\text {th }}$ Annual Meeting, April 11-14 2018.

${ }^{1}$ Department of Cardiac Surgery, University of Michigan Frankel

Cardiovascular Center, 5158 Cardiovascular Center, SPC 5864, 1500 E. Medical Center Drive, Ann Arbor, Ml 48109-5864, USA

Full list of author information is available at the end of the article
}

survival, greater demands on resources and worse long term outcomes [3, 4]. While the majority of studies have focused on the hemodynamic contributions to the right ventricular dysfunction (RVD) [5], the etiology of RVF is likely a complex interaction of mechanical burden with underlying ventricular biology.

There is gathering evidence to implicate the immune response in heart failure pathophysiology [6]. Decreased chemokine receptor expression has been associated with a greater incidence of right heart failure following durable mechanical circulatory support (MCS), and greater mortality at 1 year [7]. Pre-LVAD inflammatory status also has significant prognostic implications. Higher IL-6 levels post-

(c) The Author(s). 2019 Open Access This article is distributed under the terms of the Creative Commons Attribution 4.0 International License (http://creativecommons.org/licenses/by/4.0/), which permits unrestricted use, distribution, and reproduction in any medium, provided you give appropriate credit to the original author(s) and the source, provide a link to the Creative Commons license, and indicate if changes were made. The Creative Commons Public Domain Dedication waiver (http://creativecommons.org/publicdomain/zero/1.0/) applies to the data made available in this article, unless otherwise stated. 
LVAD implantation is associated with longer ICU stay, hospitalization and higher Sequential Organ Failure Assessment (SOFA) score after 1 week correlating with greater multi-organ dysfunction [8]. Postoperatively, elevated IL-6 levels during the early post-LVAD implant period was associated with death from multi-organ dysfunction, and monocyte activation was proposed as a critical mechanism [9].

While these cytokine and chemokine markers of inflammation are important for prognosis, they have limited availability in a clinical setting. There is also scarce long term follow up data associated with these inflammatory markers. Therefore, this study examines the white blood count (WBC) and C-reactive protein (CRP), two commonly measured lab values, to determine their prognostic value for patients undergoing durable LVAD implantation.

\section{Methods}

\section{Patients}

This study was approved by the University of Michigan Institutional Review Board (IRB\#HUM00135533). A waiver of informed consent was approved by the University of Michigan IRB. We conducted a retrospective review of prospectively collected data from 489 total patients from the University of Michigan Mechanical Circulatory Support Registry (IRB\#HUM00020274) who underwent continuous flow LVAD (cfLVAD) implantation at the from January 1, 2003 to June 1, 2017. We separated patients into a preoperative groups of low WBC $(<10.5 \mathrm{~K} / \mathrm{uL}$, $n=362)$ and high WBC $(\geq 10.5 \mathrm{~K} / \mathrm{uL}, n=127)$. We excluded patients who underwent pulsatile durable LVADs in our study since these have been largely replaced by pulsatile flow devices and carry a very different postoperative outcomes and complication profile. We included only patients aged 18 years or older and also included patients who needed postoperative biventricular support.

\section{Follow up}

Survival and heart transplant data was available for all 489 patients in this study who underwent cfLVAD implantation. These were obtained through detailed prospective clinical follow-up. Censoring was performed at the time of heart transplantation or device explant (without replacement) for myocardial recovery or device complication or device inactivation (device not removed). Longest follow-up was 12.8 years with a total follow up of 996.8 patient years. Median follow up was $1.32(\mathrm{IQR}=2.25)$ years with a mean follow up of $2.0 \pm 2.1$ years.

Right ventricular failure defined as a central venous pressure $>18 \mathrm{mmHg}$ with a cardiac index $<2.01 / \mathrm{min} / \mathrm{m} 2$ in the absence of tamponade, ventricular arrhythmias or pneumothorax requiring RVAD or inhaled pulmonary vasodilator (e.g. nitric oxide) or inotropic therapy for $>1$ week at any time following LVAD implantation [10]. Only postoperative RVF occurring during the index hospitalization following
LVAD implantation was considered. Device infection is per criteria established by the Interagency Registry for Mechanically Assisted Circulatory Support (INTERMACS) [11]. Briefly, cutaneous site infections are defined as pain, erythema or purulent drainage at the driveline site with positive skin cultures. LVAD infection are identified with positive blood cultures combined with fever and leukocytosis not explained by other potential causes.

\section{Statistical methods}

The Pearson chi square test or Fisher's exact test was used to analyze categorical variables. Student's t-test was used to compare continuous variables. Uni- and multivariable correlation with leukocytosis was determined using linear and binary logistic regression. Kaplan-Meier Survival analysis with Mantel-Cox statistics was used to analyze survival data. Statistical analysis was performed using the Statistical Package for the Social Sciences software (SPSS Inc., Chicago, IL).

\section{Results \\ Preoperative laboratory parameters and patient demographics}

Patients with preoperative $\mathrm{WBC} \geq 10.5 \mathrm{~K} / \mu \mathrm{L}$ had a mean WBC of $13.20 \pm 3.56$ and those with $\mathrm{WBC}<10.5 \mathrm{~K} / \mu \mathrm{L}$ had a mean $\mathrm{WBC}$ of $7.38+1.65$ (Table $1, P<0.001$ ). The high WBC group also had a higher level of another inflammatory marker CRP $(P<0.001)$. The lower serum $\mathrm{Na}^{+}$in this group $(P=0.004)$ was also consistent with a worse degree of heart failure. Greater hepatic impairment was also observed in the in the high WBC group along with a higher total bilirubin $(P=0.005)$, PT $(P=0.002)$ and INR $(P=0.013)$. No difference in preoperative serum creatinine $(P=0.273)$ or mixed venous oxygen saturation $(P=0.953)$ was seen (Table 1$)$. Interestingly, the high WBC group was younger $(51.21 \pm 13.01$ vs $56.53 \pm 12.96, P<0.001)$ and had a higher BMI $(P=0.005)$. No differences were seen in other demographic variables or comorbidities $(P>0.05)$ although there was a trend toward greater use of preoperative CRT-D in the high $\mathrm{WBC}$ group $(P=0.063)($ Table 1$)$.

\section{Cardiac and hemodynamic characteristics}

The high WBC group (Table 2) had a higher heart rate $(94.91 \pm 19.11$ vs $86.13 \pm 16.72, P<0.001 \mathrm{bpm})$, right atrial pressure $(9.84 \pm 5.29$ vs $8.63 \pm 4.88 \mathrm{mmHg}, P=0.019)$ and a lower right ventricular stroke work index $(551.5 \pm 226.7$ vs $615.5 \pm 269.1 \mathrm{~g} / \mathrm{m} / \mathrm{m}^{2} /$ beat, $\left.P=0.011\right)$. The high WBC group had a higher acuity INTERMACS profile (Table 2 ). There was no difference in left ventricular ejection fraction, right ventricular function or valvular characteristics between the 2 groups $(P>0.05)$. The high WBC group (Table 3) was more likely to be bridged to transplant (70.1\%) while the low WBC group was more likely to be destination therapy $(41.7 \%, P=0.019)$. Patients in the high 
Table 1 Patient Demographics and Preoperative Laboratory Results

\begin{tabular}{|c|c|c|c|}
\hline & $\mathrm{WBC}<10.5 \mathrm{~K} / \mu \mathrm{L}(n=362)$ & $\mathrm{WBC} \geq 10.5 \mathrm{~K} \mu \mathrm{L}(n=127)$ & $P$ Value \\
\hline Age (years) & $56.53 \pm 12.96$ & $51.21 \pm 13.01$ & $<0.001$ \\
\hline Male & $284(78.5 \%)$ & 99 (78.0\%) & 0.906 \\
\hline Height (cm) & $174.79 \pm 9.76$ & $173.50 \pm 10.13$ & 0.205 \\
\hline Weight (kg) & $84.72 \pm 20.87$ & $87.91 \pm 23.66$ & 0.154 \\
\hline BMI & $27.61 \pm 5.99$ & $29.62 \pm 9.04$ & 0.005 \\
\hline Hypertension & $179(49.4 \%)$ & $54(42.5 \%)$ & 0.179 \\
\hline Diabetes & $125(34.5 \%)$ & $41(32.3 \%)$ & 0.645 \\
\hline Stroke or TIA & $44(12.2 \%)$ & $16(12.6 \%)$ & 0.896 \\
\hline Carotid Disease & $27(7.5 \%)$ & $8(6.3 \%)$ & 0.663 \\
\hline Hyperlipidemia & $222(61.3 \%)$ & 71 (55.9\%) & 0.284 \\
\hline Atrial Fibrillation & $81(22.4 \%)$ & $28(22.0 \%)$ & 0.939 \\
\hline Dialysis & $0(0.0 \%)$ & $1(0.8 \%)$ & 0.091 \\
\hline ICD & $305(84.3 \%)$ & $100(78.7 \%)$ & 0.156 \\
\hline CRT-D & $180(52.5 \%)$ & $53(42.7 \%)$ & 0.063 \\
\hline WBC (K/uL) & $7.38+1.65$ & $13.20+3.56$ & $<0.001$ \\
\hline CRP (mg/dL) & $2.80+4.05$ & $5.29+6.04$ & $<0.001$ \\
\hline $\mathrm{Hb}(\mathrm{g} / \mathrm{dL})$ & $11.79+8.14$ & $11.22+2.18$ & 0.433 \\
\hline $\mathrm{Na}(\mathrm{mmol} / \mathrm{L})$ & $134.30+4.39$ & $132.95+4.92$ & 0.004 \\
\hline $\mathrm{Cl}(\mathrm{mmol} / \mathrm{L})$ & $99.28+5.69$ & $96.24+6.29$ & $<0.001$ \\
\hline HCO3 (mmol/L) & $27.72+3.76$ & $28.76+4.39$ & 0.010 \\
\hline BUN (mg/dL) & $29.73+13.90$ & $34.58+17.17$ & 0.002 \\
\hline $\mathrm{Cr}(\mathrm{mg} / \mathrm{dL})$ & $1.31+0.51$ & $1.37+0.50$ & 0.273 \\
\hline BNP (pg/mL) & $911.52+996.64$ & $983.53+1034.26$ & 0.493 \\
\hline Alk Phos (IU/L) & $100.40+43.96$ & $102.63+59.18$ & 0.655 \\
\hline Total Bili (mg/dL) & $1.11+0.67$ & $1.43+1.21$ & 0.005 \\
\hline PT (seconds) & $11.61+1.11$ & $12.05+1.88$ & 0.002 \\
\hline INR & $1.11+0.13$ & $1.16+0.20$ & 0.013 \\
\hline Uric Acid (mg/dL) & $8.36+2.90$ & $9.05+3.17$ & 0.035 \\
\hline SVO2 (\%) & $55.65+8.55$ & $55.59+9.52$ & 0.953 \\
\hline
\end{tabular}

Continuous variables are mean \pm standard deviation

WBC group were more likely to be on preoperative temporary circulatory support (TCS, $17.3 \%$ vs $6.4 \%, P<0.001$ ) and be on it for a longer period of time (6 vs 4 days, $P=0.022)$. Similarly, the high $\mathrm{WBC}$ group had a trend for more IABP (55.9\% vs $47.2 \%, P=0.093)$, and also for a longer duration (median of 2 vs 1 days, $P=0.012$ ). Multivariable analysis identified an independent association between increased WBC preoperatively with increased LDH $\quad(R=0.389, \quad P<0.001), \quad$ heart rate $(R=0.243$, $P<0.001), \quad \mathrm{CRP} \quad(\mathrm{R}=0.345, \quad P=0.006), \quad$ creatinine $(R=0.119, P=0.048)$, and INR $(R=0.164, P=0.049)$.

\section{Operative characteristics and postoperative outcomes}

Approximately a quarter of the patients underwent redo-sternotomy with no difference in redo-sternotomies between the 2 groups $(P=0.220$, Table 4$)$. There was no difference in the incidence of concomitant procedures, duration of $\mathrm{CPB}$, or LVADs $(P>0.05)$. There was no difference in the distribution of valvular regurgitant pathology between the 2 groups (Table 3 ). There was also no difference in concomitant valvular operations in the 2 groups (Table $4, P>0.4$ ). Mitral valve intervention was uncommon in our practice as its utility has remained unclear. Aortic valve intervention was performed for moderate to severe AI. Tricuspid valve procedures to improve competence was used for moderate to severe TR. In the low and high WBC groups, the proportion of patients with moderate or greater TR was 42.3 and $44.9 \%$ respectively. Moderate TR in the low and high WBC group were 28.1 and $33.9 \%$ respectively. For the high and low WBC groups respectively, there were no differences in the proportion of intrapericardial (55 (43.3) vs $143(39.5 \%))$ or 
Table 2 Preoperative Hemodynamics and INTERMACS Class

\begin{tabular}{|c|c|c|c|}
\hline & $\mathrm{WBC}<10.5 \mathrm{~K} / \mu \mathrm{L}(n=362)$ & $\mathrm{WBC} \geq 10.5 \mathrm{~K} \mu \mathrm{L}(n=127)$ & $P$ Value \\
\hline HR (bpm) & $86.13 \pm 16.72$ & $94.91 \pm 19.11$ & $<0.001$ \\
\hline Mean Arterial BP (mmHg) & $74.96 \pm 8.84$ & $76.38 \pm 9.97$ & 0.133 \\
\hline $\mathrm{CO}(\mathrm{L} / \mathrm{min})$ & $4.49 \pm 1.18$ & $4.69 \pm 1.49$ & 0.121 \\
\hline $\mathrm{Cl}\left(\mathrm{L} / \mathrm{min} / \mathrm{m}^{2}\right)$ & $2.25 \pm 0.55$ & $2.32 \pm 0.67$ & 0.291 \\
\hline PCWP (mmHg) & $19.74 \pm 6.72$ & $20.85 \pm 7.35$ & 0.122 \\
\hline PAP (mmmHg)) & $31.50 \pm 8.14$ & $32.58 \pm 8.39$ & 0.202 \\
\hline RAP $(\mathrm{mmHg})$ & $8.63 \pm 4.88$ & $9.84 \pm 5.29$ & 0.019 \\
\hline PVR (Wood Units) & $2.78 \pm 1.38$ & $2.84 \pm 1.69$ & 0.704 \\
\hline SVR (dynes/sec/cm5) & $1265.88 \pm 392.30$ & $1253.54 \pm 478.32$ & 0.776 \\
\hline TPG $(\mathrm{mmHg})$ & $11.76 \pm 4.83$ & $11.70 \pm 4.81$ & 0.899 \\
\hline RVSWI (gm/m/m²/beat) & $615.46 \pm 269.14$ & $551.54 \pm 226.69$ & 0.011 \\
\hline CVP/PCWP Ratio & $0.44 \pm 0.23$ & $0.48 \pm 0.25$ & 0.124 \\
\hline \multicolumn{4}{|l|}{ INTERMACS } \\
\hline 1 & 37 (10.2\%) & $35(27.6 \%)$ & $<0.001$ \\
\hline 2 & $90(24.9 \%)$ & $39(30.7 \%)$ & 0.198 \\
\hline 3 & 188 (51.9\%) & $40(31.5 \%)$ & $<0.001$ \\
\hline 4 & 47 (13.0\%) & $13(10.2 \%)$ & 0.417 \\
\hline
\end{tabular}

Continuous variables are mean \pm standard deviation

preperitoneally (72 (56.7\%) vs $219(60.5 \%))$ placed LVADs $(P=0.452)$.

The high WBC group (Table 5) had a longer length of intensive care unit (ICU, $P=0.046$ ) and a trend toward longer total hospital stay $(P=0.064)$. There was more postoperative renal failure requiring dialysis in the high WBC group $(P=0.014)$, but no difference in subsequent permanent dialysis $(P=0.611)$. Postoperatively (Table 5$)$, the higher WBC was more likely to experience right heart failure $(13.4 \%$ vs $5.8 \%, P=0.006$ ), and need for a right ventricular assist device (RVAD) $(11.0 \%$ vs $4.4 \%, P=0.008)$. Patients experiencing postop RVF had a higher preop WBC $(11.3 \pm 5.7$ vs $8.7 \pm 3.1)$ and CRP $(5.6 \pm 4.4$ vs $3.3 \pm 4.7)$ levels. For those who needed an RVAD, there was no difference in the duration of use $(P=0.452)$. There was also no difference in the incidence $(P=0.950)$ and duration $(P=0.514)$ of nitric oxide use (Table 5$)$. After excluding patients who underwent preoperative TCS, patients who had postoperative RVF had a higher preoperative $\mathrm{WBC}(10.3 \pm 4.4$ vs $8.5 \pm 2.7, P=0.002)$ and CRP $(5.3 \pm 4.3$ vs $2.9 \pm 4.2, \bar{P}=0.014)$ compared to those who did not. We performed a "Receiver Operating Characteristics" analysis and shows that WBC and CRP was able to predict RVF with a C-statistics of $0.661(P=0.001)$ and 0.727 $(P<0.001)$ respectively. There was no difference in other complications or operative mortality $(P>0.05)$. The high WBC group had poorer mid-term survival (Fig. 1, $P=0.042$ )

Table 3 Preoperative Echocardiographic Findings, and Mechanical Support

\begin{tabular}{llll}
\hline & WBC $<10.5 \mathrm{~K} / \mu \mathrm{L}(\mathrm{n}=362)$ & WBC $\geq 10.5 \mathrm{~K} / \mu \mathrm{L}(\mathrm{n}=127)$ & $P$ Value \\
\hline LVEF (\%) & $15.33 \pm 5.78$ & $15.40 \pm 5.60$ & 0.900 \\
Moderate-Severe RVD & $182(50.3 \%)$ & $73(57.3 \%)$ & 0.162 \\
Severe AI & $14(3.9 \%)$ & $2(1.6 \%)$ & 0.211 \\
Severe MR & $123(34.0 \%)$ & $40(31.5 \%)$ & $14(11.0 \%)$ \\
Severe TR & $53(14.6 \%)$ & $89(70.1 \%)$ & 0.610 \\
Bridge to Txp & $211(58.3 \%)$ & $38(29.9 \%)$ & 0.308 \\
Destination & $151(41.7 \%)$ & $22(17.3 \%)$ & 0.019 \\
TCS & $23(6.4 \%)$ & $6.0(\mathrm{IQR}=4.0)$ & 0.019 \\
TCS Duration (median) & $4.0(\mathrm{IQR}=2.0)$ & $71(55.9 \%)$ & $<0.001$ \\
IABP & $171(47.2 \%)$ & $2.0(\mathrm{IQR}=3.0)$ & 0.022 \\
IABP Duration (median) & $1.0(\mathrm{IQR}=1.0)$ & 0.093 \\
\hline
\end{tabular}

$\mathrm{LVEF}$ is expressed as mean \pm standard deviation. Other continuous variables were expressed as median with interquartile range 
Table 4 Operative Procedures and Parameters

\begin{tabular}{llll}
\hline & WBC $<10.5 \mathrm{~K} / \mu \mathrm{L}(n=362)$ & WBC $\geq 10.5 \mathrm{~K} / \mu \mathrm{L}(n=127)$ & $P$ Value \\
\hline Redo-Sternotomy & $103(28.5 \%)$ & $29(22.8 \%)$ & 0.220 \\
Cardiopulmonary Bypass (min) & $83.96 \pm 31.61$ & $87.65 \pm 35.348$ & 0.273 \\
Sternum Closed & $213(58.8 \%)$ & $82(64.6 \%)$ & 0.211 \\
Chest Open (median days) & $1.0(\mathrm{IQR}=1.0)$ & $1.0(\mathrm{QQR}=1.0)$ & 0.808 \\
Concomitant Surgery & & & $54(42.5 \%)$ \\
$\quad 161(44.5 \%)$ & $6(4.7 \%)$ & 0.702 \\
$\quad$ Valve procedure & $27(7.5 \%)$ & $49(38.6 \%)$ & 0.291 \\
AV procedure & $143(39.5 \%)$ & $0(0.0 \%)$ & 0.855 \\
TV Procedure & $2(0.6 \%)$ & $55(43.3 \%)$ & 0.401 \\
MV Procedure & $143(39.5 \%)$ & $72(56.7 \%)$ & 0.452 \\
Intrapericardial LVAD & $219(60.5 \%)$ & 0.452 \\
Preperitoneal LVAD & &
\end{tabular}

Cardiopulmonary bypass time was expressed as mean \pm standard deviation. Chest open days is expressed as median with interquartile range

with survival between the 2 groups diverging at approximately 2 years after cfLVAD implantation.

\section{Discussion}

Our studies demonstrate that in patients undergoing cfLVAD implantation, those who have a higher WBC preoperatively had a poorer postoperative and long term prognosis. The CRP, another commonly measured lab value, was also elevated consistent with a proinflammatory environment. While there was no difference in creatinine, there were laboratory evidence of liver dysfunction with elevations in total bilirubin and INR in the high WBC group. Higher WBC was also associated with a greater severity of heart failure with a greater proportion of patient classified as INTERMACS Patient Profile 1, higher heart rate, lower right ventricular stroke work index, as well as a greater incidence and duration of preoperative TCS. The high WBC group may be younger because more of these patients presented with acute cardiogenic shock and received temporary TCS with subsequent LVAD as bridge to transplant. Conversely, the older group were more likely to have chronic heart failure treated with destination therapy LVAD without the acute shock presentation requiring TCS. Furthermore, younger patient are more likely to mount a greater inflammatory response to

Table 5 Postoperative Outcomes

\begin{tabular}{llll}
\hline & WBC $<10.5 \mathrm{~K} / \mu \mathrm{L}(n=362)$ & WBC $\geq 10.5 \mathrm{~K} / \mu \mathrm{L}(n=127)$ & $P$ Value \\
\hline Total ICU LOS (mean days) & $10.95 \pm 17.22$ & $15.73 \pm 34.92$ & 0.046 \\
Total LOS (mean days) & $26.06 \pm 20.15$ & $30.86 \pm 35.58$ & 0.064 \\
Total Days Readmit (mean days) & $38.14 \pm 54.11$ & $31.94 \pm 45.47$ & 0.248 \\
Device Infection & $95(26.2 \%)$ & $26(20.5 \%)$ & $7(5.5 \%)$ \\
Device Exchange Infection & $21(5.8 \%)$ & $1(0.8 \%)$ & 0.195 \\
Late AV Intervention & $5(1.4 \%)$ & $32(25.2 \%)$ & 0.904 \\
Stroke & $94(26.0 \%)$ & $28(22.0 \%)$ & 0.601 \\
Hemolysis & $87(24.0 \%)$ & $11(8.7 \%)$ & 0.864 \\
Postop Dialysis/CWH & $12(3.3 \%)$ & $3(2.4 \%)$ & 0.650 \\
Postop Perm Dialysis & $6(1.7 \%)$ & $41(32.3 \%)$ & 0.014 \\
LVAD Death & $107(29.6 \%)$ & $42(33.1 \%)$ & 0.611 \\
Transplant & $132(36.5 \%)$ & $8(6.3 \%)$ & 0.565 \\
Operative Mortality & $16(4.4 \%)$ & $17(13.4 \%)$ & 0.492 \\
RV Failure & $21(5.8 \%)$ & $14(11.0 \%)$ & 0.399 \\
RVAD & $16(4.4 \%)$ & $20.50(\mathrm{IQR}=74.0)$ & 0.006 \\
Duration of RVAD (median) & $17.0(\mathrm{IQR}=18.0)$ & $125(98.4 \%)$ & 0.008 \\
Nitric Oxide Use & $356(98.3 \%)$ & $2.0(\mathrm{IQR}=2.0)$ & 0.452 \\
Nitric Duration (median days) & $2.0(\mathrm{IQR}=2.0)$ & 0.950 \\
\hline
\end{tabular}

Hospital stay times are expressed as mean \pm standard deviation. Duration of RVAD and nitric oxide use is expressed as median with interquartile range 


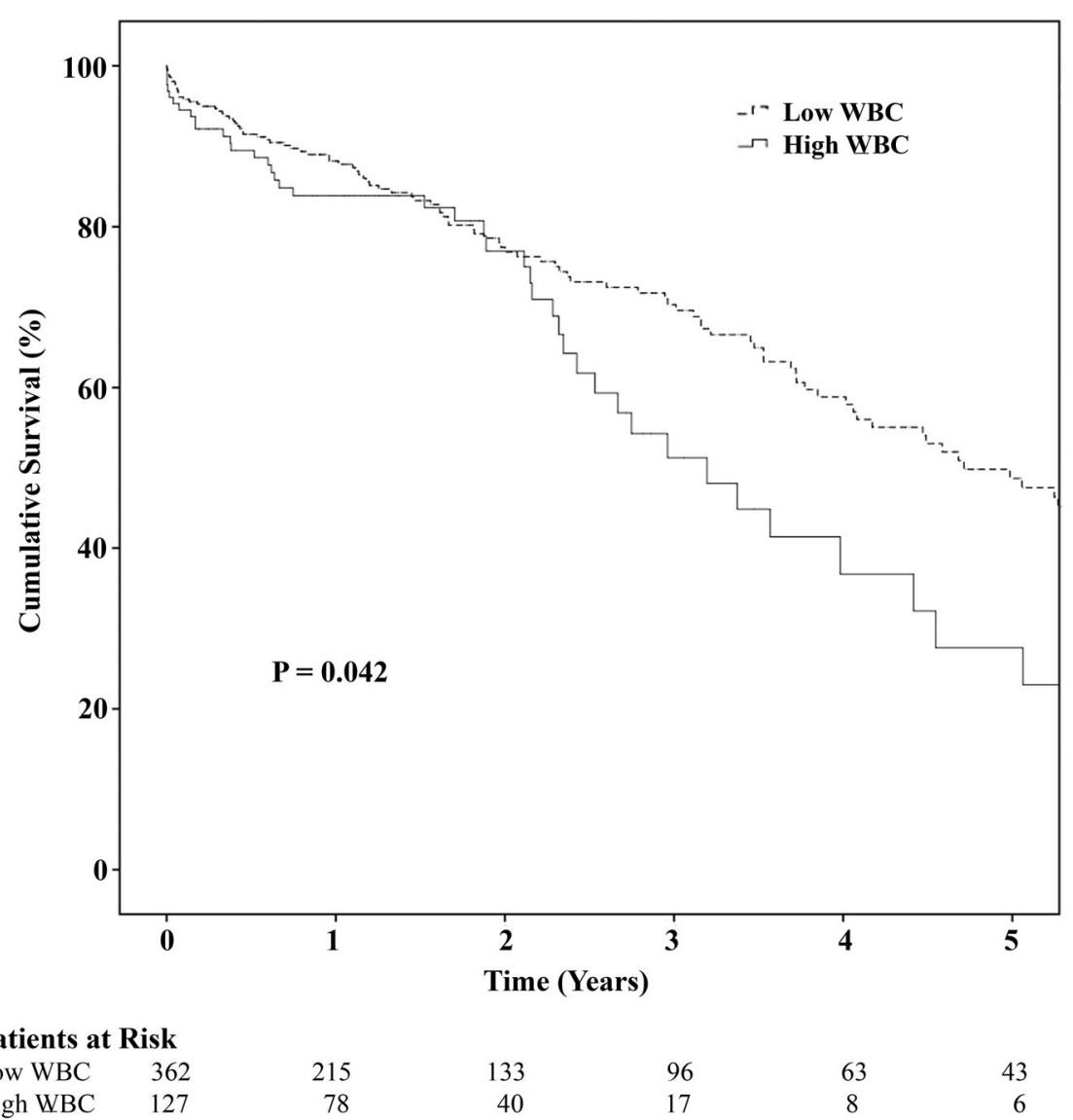

Fig. 1 Kaplan-Meier Survival for patients with low and high WBC pre-LVAD implantation

cardiovascular stress in the LVAD setting as immunosenescence and dysfunction of the immune system progresses with aging [12].

The higher WBC may be attributed to the greater underlying acuity with likely more inotropic support as well as need for TCS preoperatively. Blood contact with artificial surfaces during pre-cfLVAD mechanical support induces an increase in chemokines and cytokines such as IL-8, MCP-1, MIP-1 $\beta$, IP-10, GM-CSF [13]. This elevated preoperative inflammatory milieu in our study population is correlated with a greater incidence of RVF $(5.8 \%$ vs $13.4 \%, P=0.006)$ and need for RVAD (4.4\% vs $11.0 \%)$ postoperatively. Cytokines such as TNF- $\alpha$ and its receptor has been shown to be upregulated in the failing myocardium [14]. Its role in causing myocardial dysfunction has been demonstrated where TNF-receptor-associated factor 2 (TRAF2) expression lead to heart failure, and this can be reversed after removal of inflammatory stimulus [15]. Furthermore, increased TNF- $\alpha$ levels are associated with greater nonsurgical bleeding following LVAD implantation [16].

Many previous studies have focused on postoperative inflammatory status on prognosis. Inflammatory cytokines were higher in nonsurvivors over 30 days post-LVAD implantation. In particular TNF- $\alpha$, IL- $1 \beta$, and IL- 8 were elevated within hours to 7 days postoperatively [17]. Our finding that the high WBC group had a higher ICU and total hospital length of stay is consistent with prior findings where patients with higher preoperative IL-6, IL-8 and neopterin levels had a longer ICU stay and a greater decline in end organ function [18]. This impact on end organ dysfunction is reflected in our study where higher preoperative $\mathrm{WBC}$ was associated with a greater incidence of postoperative dialysis.

Interestingly, the high WBC group also had a greater proportion of patients that were bridged to transplantation consistent with a more severe heart failure with likely a higher incidence of biventricular dysfunction. Furthermore, our study showed that elevation in pre-cfLVAD WBC has long terms implications with a poorer mid-term survival that becomes evident 2 years after cfLVAD implantation. Late RVF may be a possible explanation for this finding.

The association of a preoperative proinflammatory state (elevated WBC and CRP) with the occurrence of postoperative RVF provides insights into the clinical relevance of inflammation in impacting myocardial 
contractility. Given the limited predictive value of current post-LVAD RVF prediction models, preoperative inflammatory markers may need to be incorporated into the algorithm for improved accuracy. It is also possible that suppression of excessive preoperative inflammation can decrease the incidence of RVF and need for RVAD.

This study has limitations due to its retrospective nature in a single institution with inherent limitations and biases. Elevation in WBC may reflect a higher INTERMACS status with greater inotrope use, use of TCS and the stress of a greater degree of heart failure. It remains unclear whether an independent elevation in WBC reflecting a greater inflammatory status is causative for poorer postoperative and long term outcomes. We cannot definitively exclude the likelihood of infection playing a role in elevated WBC since only a minority of patients have microbiological work up to exclude this possibility. Furthermore, it is possible that steroids used to treat inflammatory cardiomyopathies (e.g. giant cell myocarditis) preoperative may have contributed to elevated WBC. While there was a difference in age, high WBC group was younger but still had a poorer survival despite this. We acknowledge that there are multiple preoperative factors that may cause elevations in WBC and CRP, this introduces heterogeneity into our study groups.

\section{Conclusions}

Our study demonstrates that a preoperative proinflammatory milieu characterized by elevations of WBC and CRP can be associated with postoperative RVF. Increased WBC was also associated with a longer length of hospital and ICU stay, a higher incidence of postoperative dialysis, as well as poorer long term patient survival. Preoperative proinflammatory stimuli likely include temporary mechanical support, catecholamines, concurrent infection, and low cardiac output syndrome with tissue ischemia. Leukocytosis is likely a marker of tissue stress and hypoperfusion. Furthermore, it is well recognized that inflammation can have a negative impact on myocardial contractility with implications for RVF after LVAD implantation.

\footnotetext{
Abbreviations

BMI: Body Mass Index; cfLVAD: Continuous Flow Left Ventricular Assist Device; CPB: Cardiopulmonary Bypass; Cr: Creatinine; CRP: C-Reactive Protein; CRT-D: Cardiac Resynchronization Therapy-Defibrillator; IABP: Intra-Aortic Balloon Pump; ICU: Intensive Care Unit; INR: International Normalized Ratio; LDH: Lactate Dehydrogenase; LVAD: Left Ventricular Assist Device; MCS: Mechanical Circulatory Support; RVAD: Right Ventricular Assist Device; RVD: Right Ventricular Dysfunction; RVF: Right Ventricular Failure; SOFA: Sequential Organ Failure Assessment; TCS: Temporary Circulatory Support; WBC: White Blood Count
}

\section{Acknowledgements}

Not applicable.

\section{Funding}

No funding was provided for this study.

\section{Availability of data and materials}

The datasets used and/or analyzed during the current study are available from the corresponding author on reasonable request.

\section{Classifications}

Left ventricular assist device; right ventricular failure; inflammation; outcomes.

\section{Authors' contributions}

FDP, KA, and PCT was involved in the conception and design, manuscript drafting, analysis and interpretation of the data, data collection, as well as critical revision for important intellectual content. JWH, MAR, AB, RH, and MP was involved in the interpretation of data as well as critical revision for important intellectual content. All authors read and approved the final manuscript.

\section{Ethics approval and consent to participate}

Study was approved by the University of Michigan Institutional Review Board (IRB\#HUM00135533).

\section{Consent for publication}

A waiver of informed consent was approved by the University of Michigan IRB (IRB\#HUM00135533).

\section{Competing interests}

The authors declare that they have no competing interests.

\section{Publisher's Note}

Springer Nature remains neutral with regard to jurisdictional claims in published maps and institutional affiliations.

\section{Author details}

${ }^{1}$ Department of Cardiac Surgery, University of Michigan Frankel Cardiovascular Center, 5158 Cardiovascular Center, SPC 5864, 1500 E. Medical Center Drive, Ann Arbor, Ml 48109-5864, USA. ${ }^{2}$ Division of Cardiovascular Medicine, University of Michigan Frankel Cardiovascular Center, Ann Arbor, $\mathrm{Ml}$, USA.

Received: 22 January 2019 Accepted: 1 April 2019

Published online: 25 April 2019

\section{References}

1. Anker SD, von Haehling S. Inflammatory mediators in chronic heart failure: an overview. Heart. 2004;90(4):464-70.

2. Mann DL. Inflammatory mediators and the failing heart: past, present, and the foreseeable future. Circ Res. 2002;91(11):988-98.

3. Argiriou $\mathrm{M}$, et al. Right heart failure post left ventricular assist device implantation. J Thorac Dis. 2014;6(Suppl 1):S52-9.

4. Kalogeropoulos AP, et al. The right ventricular function after left ventricular assist device (RVF-LVAD) study: rationale and preliminary results. Eur Heart J Cardiovasc Imaging. 2016;17(4):429-37.

5. Lampert BC, Teuteberg JJ. Right ventricular failure after left ventricular assist devices. J Heart Lung Transplant. 2015;34(9):1123-30.

6. Marchant DJ, et al. Inflammation in myocardial diseases. Circ Res. 2012; 110(1):126-44

7. Nayak A, et al. Chemokine receptor patterns and right heart failure in mechanical circulatory support. J Heart Lung Transplant. 2017;36(6):657-65.

8. Caruso R, et al. Relationship between pre-implant interleukin-6 levels, inflammatory response, and early outcome in patients supported by left ventricular assist device: a prospective study. PLoS One. 2014;9(3):e90802.

9. Masai T, et al. Hepatic dysfunction after left ventricular mechanical assist in patients with end-stage heart failure: role of inflammatory response and hepatic microcirculation. Ann Thorac Surg. 2002;73(2):549-55.

10. Topilsky $Y$, et al. Echocardiographic predictors of adverse outcomes after continuous left ventricular assist device implantation. JACC CardiovasC Imaging. 2011;4(3):211-22.

11. Simeon S, et al. Left ventricular assist device-related infections: a multicentric study. Clin Microbiol Infect. 2017;23(10):748-51.

12. Aw D, Silva $A B$, Palmer DB. Immunosenescence: emerging challenges for an ageing population. Immunology. 2007;120(4):435-46. 
13. Lappegard KT, et al. The artificial surface-induced whole blood inflammatory reaction revealed by increases in a series of chemokines and growth factors is largely complement dependent. J Biomed Mater Res A. 2008;87(1):129-35.

14. Torre-Amione $\mathrm{G}$, et al. Tumor necrosis factor-alpha and tumor necrosis factor receptors in the failing human heart. Circulation. 1996;93(4):704-11.

15. Topkara VK, et al. Functional significance of the discordance between transcriptional profile and left ventricular structure/function during reverse remodeling. JCI Insight. 2016;1(4):e86038.

16. Tabit CE, et al. Tumor necrosis factor-alpha levels and non-surgical bleeding in continuous-flow left ventricular assist devices. J Heart Lung Transplant. 2018:37(1):107-15.

17. Caruso R, et al. Early expression of pro- and anti-inflammatory cytokines in left ventricular assist device recipients with multiple organ failure syndrome. ASAIO J. 2010;56(4):313-8.

18. Caruso R, et al. Association of pre-operative interleukin-6 levels with interagency registry for mechanically assisted circulatory support profiles and intensive care unit stay in left ventricular assist device patients. J Heart Lung Transplant. 2012;31(6):625-33.

Ready to submit your research? Choose BMC and benefit from:

- fast, convenient online submission

- thorough peer review by experienced researchers in your field

- rapid publication on acceptance

- support for research data, including large and complex data types

- gold Open Access which fosters wider collaboration and increased citations

- maximum visibility for your research: over $100 \mathrm{M}$ website views per year

At $\mathrm{BMC}$, research is always in progress.

Learn more biomedcentral.com/submissions 